\title{
Study of interstitial lung diseases at a tertiary care center
}

\author{
Ramakrishna Rachakonda ${ }^{1, *}$, Nandeeswara Reddy $\mathbf{C V}^{2}$ \\ ${ }^{1}$ Professor, NRI Medical College, Guntur, Andhra Pradesh, ${ }^{2}$ Senior Resident, Katuri Medical College, Guntur, Andhra Pradesh, \\ India
}

*Corresponding Author:

Email: ramakrishna45@yahoo.co.in

\begin{abstract}
Introduction: Interstitial Lung Diseases are frequent causes of morbidity and mortality and are frequently diagnosed because of the usage of High Resolution Computerised Tomography (HRCT). Interstitial Lung Diseases (ILDs) result from known and unknown causes and result in variable amount of interstitial and pulmonary parenchymal inflammation and fibrosis.

Material and Methods: A total of 104 patients diagnosed on the basis of clinical and radiological criteria as interstitial Lung disease attending Katuri Medical College Hospital, Guntur from June 2015 to June 2018 above 18 years of age were taken in to the study.

Results: Predominant interstitial lung disease was idiopathic interstitial fibrosis (IPF) (46\%). Non-IPF interstitial lung diseases constituted $56 \%$. Females outnumbered males in our study with a ratio of 1.7:1. Mean age of the ILD patients was 50.19. Cough and breathlessness were universal in our ILD patients. There are equal number of smokers and nonsmokers. > 75\% of patients presented with Grade II MMRC or more. $75 \%$ of our patients of ILD desaturated on 6 minute walk testing. PFT revealed restrictive pattern in $69 \%$, mixed pattern in $26.92 \%$ and obstructive pattern in $4 \% .50 \%$ of patients had DLCO of more than $60 \%$. Smoking history correlated with decrease in DLCO. BAL fluid analysis suggested neutrophilic preponderance in $42 \%$ and lymphocytic preponderance in 58\%.Basing on the clinical and radiological criteria IPF was diagnosed in 46\%, NSIP in $21 \%$, NSIP CTD in 12\% and Hypersensitivity Pneumonitis in 14\%. 62.5\% had associated pulmonary arterial hypertension. Smoking history correlated with presence of PAH.

Conclusions: ILDs are common in clinical practice. Diagnosed predominantly after the frequent usage of HRCT in symptomatic patients. History analysis, clinical examination, chest X-ray, bronchoscopy, bronchoalveolar lavage (BAL), pulmonary Function Testing, six minute walk test, echocardiography, carbon dioxide diffusion capacity (DLCO) testing help in the diagnosis.
\end{abstract}

Keywords: Desaturation; Sarcoidosis; Hypersensitivity pneumonitis; Pulmonary arterial hypertension.

\section{Introduction:}

The interstitial lung diseases are a clinically challenging and diverse group of disorders characterized by varying degrees of fibrosis and inflammation of the lung parenchyma or interstitium. The interstitial lung diseases have similar symptoms, similar radiological presentation. More than 150 interstitial lung diseases with known or unknown etiology have been identified. Occupational lung diseases, drug induced lung diseases, hypersensitivity pneumonitis, granulomatous lung disease, lung diseases associated with exposure to organic and inorganic dusts and idiopathic interstitial pneumonias share the common signs and symptoms of interstitial lung diseases associated with variable amount of interstitial and pulmonary fibrosis. For each ILD there may be an acute phase followed by a phase of chronicity with acute exacerbation. Among ILDs of known cause, the largest group includes diseases due to inhalation of inorganic dusts, organic dusts and various irritant and noxious gases. The number of ILDs of unknown cause is also very large. The major ones are Idiopathic Pulmonary Fibrosis (IPF), Sarcoidosis, Nonspecific Interstitial Pneumonitis (NSIP), ILD associated with collagen vascular diseases (CTD-ILDs). Time of diagnosis differs between the ILD diseases, ranging from the fourth decade for sarcoidosis to the sixth decade for IPF. Some interstitial lung diseases like hypersensitivity pneumonitis and connective tissue disease associated ILD are diagnosed in patients less than 50 years of age [1]. A multi disciplinary approach is necessary in the diagnosis of interstitial lung diseases. In view of the comorbidities it is not always possible to do a biopsy [2].

Among the ILDs idiopathic pulmonary fibrosis is the most lethal entity and shows high heterogeneity in clinical behavior [3]. IPF is the commonest idiopathic ILD and IPF represents nearly $30 \%$ of the incident case. The disease process extends into alveolar spaces, acini, bronchiolar lumen and bronchioles [2,4].

\section{Aims and Objectives:}

To study the clinical profile, the radiological profile (chest X-ray and HRCT Scan), spirometry profile, BAL fluid analysis, results of 6 minute walk test values, DLCO, echocardiography findings in patients of ILD and analyze the results and compare with the severity of ILDs.

\section{Material and Methods:}

The present study comprises of 104 patients who are admitted in the Department of Pulmonary Medicine, Katuri Medical College, Guntur, from November 2015 to June 2018. 
Study design: It is a prospective study consisting of 104 patients examining the clinical, radiological and pulmonary function test profile

Inclusion criteria: Patients having symptoms suggestive of interstitial lung disease by clinical, radiological and extrapulmonary symptoms above 18 years of age are included in the study. Patients with known connective tissue disease with respiratory symptoms referred to the department of Pulmonary medicine of Katuri Medical College, Guntur are taken in to the study.

Exclusion criteria: ILD like infections eg. miliary tuberculosis and pneumocystis jirovecii pneumonia (PJP). 2. ILD like malignancies eg. lymphangitis carcinomatosis 3.pulmonary kochs co-existing with ILD. 4. Patients of age less than 18 yrs. 5. Patients having serious interstitial disease with resting hypoxia requiring ICU admission and moribund patients are excluded from the study

\section{Study protocol}

Prior to the investigation hospital ethical committee approval was taken and consent from the individual patients were taken.

Among a total of 104 patients of interstitial lung disease a detailed history of symptoms, age, sex, occupation and habits like smoking were taken. Clinical symptoms like cough, haemoptysis, breathlessness, loss of appetite, loss of weight, and clinical signs were evaluated in all patients. Baseline Chest X-ray both PA (Postero-anterior view, Base line blood investigations like Haemoglobin, TLC, DLC, ESR, peripheral smear, blood urea, serum creatinine, random blood Sugar, serum bilirubin, BT,CT were done. Mantoux, sputum for AFB, sputum for malignant cytology, sputum for gram stain and Culture \& Sensitivity were done. Patients were screened for HIV and HBsAg. HRCT Chest and FOB were done. BAL fluid analyses for total leukocyte count (TLC), Differential leukocyte count (DLC) were sent. Pulmonary function tests like Spirometry, 6 minute walk test and DLCO were done. Radiological assessment was done in all patients. The diagnosis of interstitial lung disease was established by HRCT or Chest X Ray PA view correlating with history and clinical examination.

Results were analyzed with minitab 17 software and results verified. DLCO and PAH results were correlated with smoking history and Chi-square values and p-values were calculated for statistical significance.

\section{Observations and Results:}

Among 104 patients of interstitial lung disease, 48 patients $(46 \%)$ are diagnosed as IPF and 56 patients $(54 \%)$ are diagnosed as non IPF.

\section{Sex distribution}

In the present study total number of patients are 104 and among them $38(36.5 \%)$ were males and $66(63.5 \%)$ were females. Male: female ratio is 1:1.7.

\section{Age distribution}

The mean age of patients in this study was 50.19 years. Most of the patients were in 56-65 years age group.

Table 1: Age and sex distribution of patients

\begin{tabular}{|l|c|c|c|}
\hline Age (years) & Male & Female & Percentage \\
\hline $16-25$ & 0 & 4 & $1.92 \%$ \\
\hline $26-35$ & 6 & 4 & $9.6 \%$ \\
\hline $36-45$ & 6 & 26 & $30.76 \%$ \\
\hline $46-55$ & 8 & 12 & $19.2 \%$ \\
\hline $56-65$ & 16 & 22 & $36.53 \%$ \\
\hline $66-75$ & 0 & 0 & $0 \%$ \\
\hline $76-85$ & 0 & 0 & $0 \%$ \\
\hline $86-95$ & 2 & 0 & $1.92 \%$ \\
\hline
\end{tabular}

Table 2: Symptomatic analysis of patients of Interstitial Lung disease

\begin{tabular}{|l|c|}
\hline Symptoms & Percentage \\
\hline Cough & $100 \%$ \\
\hline Breathlessness & $100 \%$ \\
\hline Loss of weight & $15.38 \%$ \\
\hline Loss of appetite & $15.38 \%$ \\
\hline Haemoptysis & 3.84 \\
\hline
\end{tabular}

Table 3: Shortness of breathlessness --- Grade of MMRC

\begin{tabular}{|c|c|c|}
\hline $\begin{array}{l}\text { Grade of } \\
\text { MMRC }\end{array}$ & $\begin{array}{l}\text { No. of } \\
\text { patients }\end{array}$ & Percentage \\
\hline I & 24 & $23 \%$ \\
\hline II & 48 & $46.15 \%$ \\
\hline III & 22 & $21.15 \%$ \\
\hline IV & 10 & $9.6 \%$ \\
\hline
\end{tabular}

\section{Smoking}

In the present study of total 104 patients 52 patients were smokers and 52 Patients were non smokers.

Table 4: Smoking in males and females

\begin{tabular}{|l|c|c|}
\hline \multicolumn{1}{|c|}{ Sex } & Males & Females \\
\hline Smokers & 36 & 16 \\
\hline Nonsmokers & 2 & 50 \\
\hline
\end{tabular}

\section{Pulmonary function tests}

Observation of six minute walk test performance among patients of interstitial lung disease. Among 104 patients 78 patients $(75 \%)$ were desaturated with a decrease in SPO2 of $4 \%$ or more on six minute walk testing and 26 patients $(25 \%)$ were not desaturated spirometry. 
Table 5: Result of spirometry among patients of ILD

\begin{tabular}{|l|c|c|c|c|}
\hline $\begin{array}{c}\text { Pattern of } \\
\text { spirometry }\end{array}$ & $\begin{array}{c}\text { Number of } \\
\text { patients }\end{array}$ & Smokers & $\begin{array}{c}\text { Non } \\
\text { Smokers }\end{array}$ & $\begin{array}{c}\text { Percentage } \\
\text { of patients }\end{array}$ \\
\hline Restrictive & 72 & 28 & 44 & $69 \%$ \\
\hline Obstructive & 4 & 4 & 0 & $4 \%$ \\
\hline Mixed pattern & 28 & 20 & 08 & $26.92 \%$ \\
\hline
\end{tabular}

Though there are equal number of smokers and nonsmokers in our study of ILDs spirometric observation of mixed pattern and obstructive pattern are more among smokers and restrictive pattern is more among nonsmokers and chi-square statistic is 12.6984 and $\mathrm{p}$ value is 0.001748 and the result is statistically significant at a p-value of $<0.05$.

Table 6: DLCO Test results among patients of ILD

\begin{tabular}{|c|c|c|c|}
\hline DLCO\% & Males & Females & Total n=104 \\
\hline $30-40 \%$ & 6 & 4 & 10 \\
\hline $41-50 \%$ & 12 & 12 & 24 \\
\hline $51-60 \%$ & 2 & 18 & 20 \\
\hline $61-70 \%$ & 14 & 22 & 36 \\
\hline $71-80 \%$ & 2 & 10 & 12 \\
\hline $81-90 \%$ & 2 & 0 & 2 \\
\hline
\end{tabular}

Table 7: DLCO Test results among smokers and nonsmokers

\begin{tabular}{|l|c|c|c|}
\hline DLCO\% & $\begin{array}{c}\text { Smokers } \\
\mathbf{N = 5 2}\end{array}$ & $\begin{array}{c}\text { Non-smokers } \\
\mathbf{N = 5 2}\end{array}$ & Total \\
\hline $30-40 \%$ & 08 & 02 & 10 \\
\hline $40-50 \%$ & 18 & 06 & 24 \\
\hline $50-60 \%$ & 08 & 12 & 20 \\
\hline $60-70 \%$ & 08 & 28 & 36 \\
\hline$>70 \%$ & 10 & 04 & 14 \\
\hline
\end{tabular}

Smokers in our study have a lower DLCO compared to the non smokers and the Chi-square statistic is 24.0825 and $\mathrm{p}$-value is 0.000077 and the value is statistically significant at a p-value of $<0.05$.

Table 8: Diagnosis based on Clinical and radiological examination

\begin{tabular}{|l|c|c|}
\hline & $\begin{array}{c}\text { Number of } \\
\text { patients n=104 }\end{array}$ & Percentage \\
\hline IPF & 48 & $46 \%$ \\
\hline NSIP & 22 & $21 \%$ \\
\hline NSIP with CTD & 12 & $12 \%$ \\
\hline $\begin{array}{l}\text { Hypersensitivity } \\
\text { pneumonitis }\end{array}$ & 14 & $13 \%$ \\
\hline $\begin{array}{l}\text { Rare forms } \\
\text { (LAM,LCH) }\end{array}$ & 4 & $4 \%$ \\
\hline Drug induced & 4 & $4 \%$ \\
\hline
\end{tabular}

Table 9: Bronchoscopy and BAL fluid analysis

\begin{tabular}{|l|c|c|}
\hline \multicolumn{1}{|c|}{ BAL fluid Analysis } & $\begin{array}{c}\text { Number of } \\
\text { patients }\end{array}$ & Percentage \\
\hline Neutrophil predominant & 44 & $42.30 \%$ \\
\hline Lymphocyte predominant & 60 & $57.70 \%$ \\
\hline
\end{tabular}

Table 10: Pulmonary arterial Hypertension among Patients of ILD

\begin{tabular}{|l|l|l|}
\hline Total No. of ILD & $\begin{array}{c}\text { No. having PAH by } \\
\text { Echo }\end{array}$ & No PAH \\
\hline 104 & $65(62.5 \%)$ & $39(37.5 \%)$ \\
\hline Mild PAH & $11(16.92 \%)$ & \\
\hline Moderate PAH & $23(35.38 \%)$ & \\
\hline Severe PAH & $31(47.69 \%)$ & \\
\hline
\end{tabular}


Table 11: Correlation of PAH among smokers and Non smokers

\begin{tabular}{|l|c|c|c|c|}
\hline $\begin{array}{c}\text { No. Patients } \\
\mathbf{1 0 4}\end{array}$ & $\begin{array}{c}\text { PAH - Smoker } \\
\mathbf{4 8}\end{array}$ & $\begin{array}{c}\text { PAH } \\
\text { Nonsmoker } \\
\mathbf{1 7}\end{array}$ & $\begin{array}{c}\text { No PAH - } \\
\text { Smoker } \\
\mathbf{0 4}\end{array}$ & $\begin{array}{c}\text { NO PAH } \\
\text { Nonsmoker } \\
\mathbf{3 5}\end{array}$ \\
\hline Type of PAH & 48 & 17 & 04 & 35 \\
\hline Mild & 08 & 03 & & \\
\hline Moderate & 16 & 07 & & \\
\hline Severe & 24 & 07 & & \\
\hline
\end{tabular}

Severity of Pulmonary artery Hypertension is correlated with the smoking status by Chi-square test. The Chisquare statistic is 0.4303 . p-value is 0.806417 and the value is not statistically significant at a p-value of $<0.05$

Table 12: Smoking status correlated with presence of PAH

\begin{tabular}{|l|c|c|}
\hline \multicolumn{1}{|c|}{ PAH N=65 No PAH N=39 } & Smokers & Nonsmokers \\
\hline PAH $=65$ & 48 & 17 \\
\hline No $P A H=39$ & 04 & 35 \\
\hline
\end{tabular}

When presence or absence of PAH was correlated with the smoking status by Chi-square test the Chisquare statistic is 39.4256 and the p-value is <.00001. The value is statistically significant for a p-value of $<0.05$.

Pulmonary arterial hypertension is more among smoker ILD patients and the value is statistically significant. Severity of PAH did not correlate with the smoking status.

\section{Summary of results:}

The mean age of patients of ILD in this study is 50.19 years. $36.53 \%$ of the patients are in 56-65 years age group. Among males no ILD case was reported below 25 years in our study. $86 \%$ of ILD patients are present in 36-65 years age group. No ILDs were reported among female patients in extreme age group. Cough and breathlessness were the predominant symptoms among ILD patients and were present in all the patients. Majority of patients presented in Grade II and Grade III MMRC scale of breathlessness. There were equal number of smokers and nonsmokers among ILD patients. Among smokers $69 \%$ were male $31 \%$ female (When active smoking and significant passive smoking were taken in to consideration). $75 \%$ of the patients desaturated on 6 minute walk test with more than $4 \%$ decrease in SPO2 suggesting that the disease is advanced among the patients studied. Spirometry revealed $69 \%$ of the patients had restrictive disease and $14 \%$ both obstructive and restrictive disease. More than two thirds presented with a DLCO of more than $60 \%$.

Predominant ILD detected among 104 patients was IPF amounting to $46 \%$. NSIP was seen in $33 \%$ and half of them had associated connective tissue disease. Hypersensitivity pneumonitis both acute and chronic was frequent occurring in $13 \%$. BAL fluid revealed lymphocytic predominance in $58 \%$ and neutrophilic predominance in $42 \%$. Two thirds of the patients of ILD had pulmonary arterial hypertension and nearly half of them had severe PAH

\section{Discussion:}

Interstitial lung diseases are frequently diagnosed in clinical practice particularly after HRCT chest has been popularized. Idiopathic pulmonary fibrosis is the commonest ILD in our study followed by NSIP and NSIP associated with CTD. European studies and some Indian studies showed sarcoidosis is the commonest cause of ILD followed by IPF and Hypersensitivity Pneumonitis $[5,6]$. There is an increased risk of ILD among the patients with Rheumatoid arthritis prognosis of ILD associated with Rheumatoid arthritis is worse compared to RA patients without ILD. [3] ILDs with unknown cause always outnumber ILDs with known cause.

Indian registry of ILDs revealed sarcoidosis as the commonest cause of interstitial lung disease ( ILD), followed by idiopathic pulmonary fibrosis( IPF), connective tissue disease ( CTD) related ILD and Hhypersensitivity pneumonitis (HP). Histopathological confirmation could be obtained from $40 \%$ of cases [7]. In the Pakistani registry of ILD, interstitial pulmonary fibrosis (IPF) was the commonest cause accounting for $33 \%$ [8]. Singh S, Collins BF study had hypersensitivity pneumonitis (HP) as the commonest ILD amounting to $45 \%$, CTD-ILD in $13.9 \%$, and idiopathic pulmonary fibrosis in $13.7 \%$ [9] Srikala study from Kerala study showed IPF in $26 \%$ and non IPF in $74 \%$. HRCT was diagnostic in all patients of IPF. PAH was more common among IPF patients [10].

Our study showed female preponderance where as other studies from India and abroad showed male predominance. A multi disciplinary approach is needed in the diagnosis of ILDs. Prevalence of ILD increased with age and smoking, history of Pneumonia, COPD, male sex are associated with increased risk of ILD as according to an east Asian study [11].

More than $80 \%$ of cases in our study were diagnosed in patients between 36 and 65 years of age. Mean age in our study was 50.9 years. A Turkish study and several other studies also showed a mean age of 51.8 years. This study showed sarcoidosis as the 
commonest ILD followed by IPF [12]. Rajkumar study showed a mean age of 44.24 in their study of 289 cases with a female preponderance in nearly $55 \%$ of cases [13].

Thus various studies in India and abroad showed variable preponderance of ILD among male and female population. Our study showed a female: male ratio of 1.73:1 among ILD patients

Spirometry is important in the diagnosis of restrictive lung disease. Decreased total lung capacity (TLC) is an earlier parameter in ILDs [14]. In our study of ILD Patients spirometric observation of mixed pattern and obstructive pattern are more among smokers and restrictive pattern is more among nonsmokers and the values are statistically significant.

Evaluation of diffusing capacity of the lung for carbon monoxide normalized per liter alveolar volume as a parameter for assessment of interstitial lung diseases is very useful. Decreased FVC and exertional desaturation to $<88 \%$ are important criteria in the assessment of ILDs [15].

Smokers in our study have lower DLCO values compared to nonsmokers and the values are statistically significant. Najeeb Hassan Mohammed study from Iraq showed lower values of DLCO among smokers which correlates with our study [14]. Tobacco smoke exposure increases the incidence of Pulmonary arterial Hypertension especially among male smokers [16].

Nermine M. Riad stated that evaluation DLCO and DLCO/VA (Alveolar volume) should be interpreted coherently with each other especially in restrictive lung diseases. (Alveolar Volume)VA and TLC-SB (Single breath) give a good guide for lung volume in interstitial lung disease evaluation [17]. According to Hoda, Aliae AR Mohamed-Hussein study evaluation of FVC/DLCO ratio is a predictor for pulmonary hypertension in patients with interstitial lung diseases [18]. In Siribaddana et al., study HRCT findings do not correlate with spirometry and six minute walk performance [19]. In our study radiologically diagnosed cases of ILD presented in severe stage and three fourths of them showed desaturation on 6 minute walk test. In our study there is a statistically significant increase in the incidence of Pulmonary Arterial Hypertension among smokers compared to non-smokers. But the severity of PAH did not correlate with the smoking status.

Katerina D. Samara study showed smoking as a pathogenetic factor and causes increased pulmonary fibrosis among patients of Interstitial Lung Disease [20]. Hussein, et al. study among a total of 44 ILD patients showed mean age was 45.7 years. Male preponderance was seen. Frequent HRCT parameter was fibrotic with reticular and honey combing pattern in $45 \%$. TLC and DLCO correlated with severity of disease [21].

In Boubacar Efared study, the mean age was 52.78 years; $74.83 \%$ were women $22 \%$ had sarcoïdosis,
$15.6 \%$ had idiopathic pulmonary fibrosis (IPF), $14.18 \%$ had other idiopathic interstitial pneumonia (IIP) and 9.9\% had connective tissue disease (CTD). They observed that in the diagnosis of ILD BAL cytological analysis has a limited value and should be accompanied by other investigation [22]. Jesudason et al., study (2015) showed Symptoms, PFT and exercise testing had good correlation with HRCT. DLCO corrected \% of predicted correlated best with HRCT [24].

Pulmonary arterial Hypertension in Interstitial Lung disease is associated with increased symptoms, reduced exercise capacity and poor prognosis [24]. Sometimes pulmonary hypertension can be high out of proportion to the hypoxia and amount of parenchymal damage. Our study also showed two thirds of the patients had pulmonary arterial hypertsion and nearly half of them had severe hypertension by echocardiography [18].

\section{Conclusions:}

Interstitial Lung diseases are now frequently diagnosed because of HRCT. They cause considerable morbidity and mortality. Identification etiology and early diagnosis and treatment can prevent long term complications. Smoking correlates with low DLCO and presence of pulmonary arterial hypertension.

Conflicts of interests: The authors have no conflicts of interests with any one.

Acknowledgements: We acknowledge the cooperation of departments of Pulmonary Medicine and Radiology, Katuri Medical college, Guntur,and NRI Medical College, Chinakakani, Mangalagiri, Guntur district.

\section{References:}

1. Wallis A. The diagnosis and management of interstitial lung diseases, The BMJ. 9 May 2015

2. Chaudhuri N, Spencer L, Greaves M, Bishop P, Chaturvedi A, Leonard C. A Review of the Multidisciplinary Diagnosis of Interstitial Lung Diseases: A Retrospective Analysis in a Single UK Specialist Centre, J Clin Med 2016;5:66.

3. Antoniou KM , Margaritopoulos GA, Tomassetti S, Bonella F, Costabel Venerino Poletti U. Interstitial lung disease. Eur Respir Rev 2014;23:40-54.

4. Kumar R, Gupta N, Goel N. Spectrum of interstitial lung disease at a tertiary care centre in India, Pneumonol Alergol Pol 2014;82:218-26.

5. Raghu G, Brown KK. Interstitial lung disease: clinical evaluation and keys to an accurate diagnosis. Clin Chest Med 2004;25:409-19.

6. Mikolasch TA, Garthwaite HAS, Porter J. Update in diagnosis and management of interstitial lung disease Clinical Medicine 2016;16(6):71-8.

7. Raghu G, Mehta S. Interstitial lung disease (ILD) in India: Insights and lessons from the prospective, landmark ILD-India registry, Lung Ind 2016;33(6):58991.

8. Ansarie M, Naseem A, Kasmani A. Profile of Interstitial Lung Diseases in Pakistan, Karachi Pulmonology Clinics Registry Data: Jan 2012-Aug 2013. Chest. 2014;145:Supple, Page 241A(15). 
9. Singh S, Collins BF, Sharma BB, Joshi JM, Talwar D, Katiyar S, et al. Interstitial Lung Disease in India. Results of a Prospective Registry, Am J Respir Crit Care Med 2017;15;195(6):801-13.

10. Sreekala C, Soofiya M, Nair S, Kumari KA, Interstitial lung diseases - a tertiary care center experience. Int J Med Res Rev 2017;7:17.

11. Choi WI, Dauti S, Kim HJ, Park SH, Park JS, Lee CW. Risk factors for interstitial lung disease: a 9-year Nationwide population-based study. BMC Pulmonary Med 2018;18:96.

12. Okumus B, Uzaslan E, Akgün M, Cetinkaya E, Turan O, Akkoclu A, et al. Epidemiology and distribution of interstitial lung diseases in Turkey. Clin Respir J 2014;8:55-62.

13. Kumar R. Spectrum of interstitial lung disease at a tertiary care centrein India. Pneumonol Alergol Pol 2016;82:218-28.

14. Boros PW, Franczuk M, Wesolowski S. Value of spirometry in detecting volume restriction in interstitial lung disease patients. Spirometry in interstitial lung diseases. Respiration 2004;71(4):374-9.

15. Fernando J. Martinez and Kevin Flaherty, Pulmonary Function Testing in Idiopathic Interstitial Pneumonias, Proc Am Thorac Soc 2006;3(4):315-21.

16. Keusch S. Tobacco Smoke Exposure in Pulmonary Arterial and Thromboembolic Pulmonary Hypertension, Respiration 2014;88:38-45.

17. Riad NM, SalemHM, Diab HS. Evaluation of diffusing capacity of the lung for carbon monoxide normalized per liter alveolar volume as a parameter for assessment of interstitial lung diseases, Egypt J Bronchol 2014 8:51-6.

18. Hoda A, Mohamed-Hussein AR. Evaluation of FVC/DLCO ratio as a predictor for pulmonary hypertension in patients with interstitial lung diseases. Eur Respir J 2017;50:PA861.

19. SiribaddanaA, Muthunayake MT. Correlation between spirometry, six minute walk test and HRCT characteristics of patients with interstitial lung diseases in a tertiary care center in Sri Lanka. Eur Respir J 2012;40:P3673.

20. Samara KD. Smoking and Pulmonary Fibrosis: Novel Insights. Pulm Med 2011:461439.

21. Hussein K. Correlation of high resolution CT patterns to pulmonary function tests in patients with interstitial lung diseases, Eur Respir J 2016;34:4.

22. Efared B. The diagnostic value of the bronchoalveolar lavage in interstitial lung diseases. J Negative Results Bio Med 2017;16:4.

23. Junde I. The correlation of symptoms, pulmonary function tests and exercise testing with high-resolution computed tomography in patients with idiopathic interstitial pneumonia in a tertiary care hospital in South India, Lung Ind 2015;32(6):584-88.

24. Caminati A. Pulmonary hypertension in chronic interstitial lung diseases. Eur Respir Rev 2013;22:292301.

How to cite this article: Rachakonda R., Reddy N. Role of fiberoptic bronchoscopy in patients with respiratory complications in multispecialty intensive care units. IP Indian J Immunol Respiratory Med 2018;3(3):121-126. 